Military Technical College Kobry El-Kobbah, Cairo, Egypt

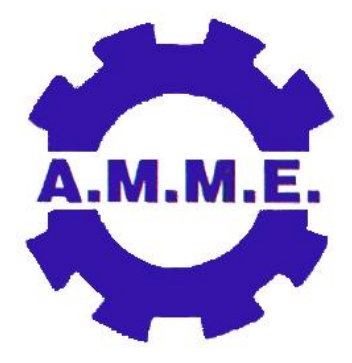

14th International Conference on

Applied Mechanics and

Mechanical Engineering.

\title{
Dynamic Snap-through of Thin-walled Spherical Shell under Impact
}

By

T.X. Yu*

D. Karagiozova**

X.W. Zhang*

\begin{abstract}
:
In order to study the dynamic effects on the snap-through behaviour of an elastic spherical shell under normal impact, ping-pong balls were accelerated by projectiles fired from an airgun and impinged onto a rigid plate with the velocity ranging $10-50 \mathrm{~m} / \mathrm{s}$. Apart from the forcedisplacement relationship, a particular attention was paid to the evolution of the contact zone between the ball and the plate, as recorded by a high-speed digital camera. As a result, the impact duration, the maximum contact diameter, and the contact diameter at snap-through buckling under different impact velocities were obtained. An axi-symmetric finite element model is generated and the dynamic simulation is conducted using ABAQUS/Explicit. Based on the experiments and simulations, a theoretical model is proposed, which simplifies the deformed shape by piecewise constant-curvature regions, but captures the major features of the deformation process of a thin-walled spherical shell, such as the onset of the snapthrough buckling, the evolution of the contact zone, etc. By taking into account the inertia effects in the deformed regions and minimizing the total work done, the evolution of the deformed shape of the shell is revealed. The theoretical predictions for the contact force and contact diameter are in good agreement, while it is concluded that the local inertia is mainly responsible for the difference between the dynamic deformation behavior and the quasi-static one.
\end{abstract}

Keywords: thin-walled spherical shell, impact, ping pong ball, snap-through buckling 
Proceeding of the $14^{\text {th }}$ AMME Conference, $25-27$ May 2010

* Department of Mechanical Engineering, The Hong Kong University of Science and Technology, Clear Water Bay, Kowloon, Hong Kong, China

** Institute of Mechanics, Bulgarian Academy of Sciences, Sofia, Bulgaria 


\section{Introduction:}

The snap-through buckling and post-buckling behavior of thin-walled spherical shells under normal compression has received considerable attention in the analyses published by Updike and Kalnins [1-2], Leckie and Penny [3], Morris and Calladine [4], Kitching et al. [5]. Three distinct stages of the deformation process, namely pre-buckling stage, symmetric and nonsymmetric post-buckling stages were identified and analysed. However, the previous investigations mostly focused on the static behaviour and load-displacement curves of the shell, while the dynamic effects under impact loading remain unclear, which are important for the characterization of the energy absorption capacity of the shell.

This paper reports our experiments, in which standard ping pong balls were chosen as specimens to study the quasi-static and dynamic snap-through of a thin-walled spherical shell compressed/impinging onto a rigid plate. The evolution of the contact region in both quasistatic and impact tests were recorded, while a special attention was paid to the dynamic effects on the process. Furthermore, a numerical simulation using FEM and a theoretical analysis based on an energy method are conducted to reveal details of the dynamic behavior of a thin-walled spherical shell.

\section{Experiment study:}

Standard ping-pong balls with outer diameter $d=39.4 \mathrm{~mm}$, thickness $h=0.38 \mathrm{~mm}$, mass $m=$ $2.6 \mathrm{~g}$ and made of celluloid were used in this study. The Young's modulus and yield stress of the material are $E=2.4 \mathrm{GPa}$ and $Y=54 \mathrm{MPa}$, respectively [6].

Quasi-static compression tests were conducted on an MTS machine with a loading speed $2 \mathrm{~mm} / \mathrm{min}$. The experimental setup is shown in Fig. 1(a); the ping pong ball was compressed onto a transparent PMMA plate and the buckling occurred only on the bottom side of the specimen. As illustrated in Fig. 1(b), during an impact test, the bullet was first shot by an air gun and then it accelerated the ping pong ball through a steel block. Subsequently, the pingpong ball flied out and impacted onto a thick PMMA plate, through which the contact region was recorded by a high speed camera with frame rate 20,000/s. A uniform layer of lubricant was spread on the surface of the PMMA plate in order to distinguish the contact region from surrounding areas. The impact velocity and contact region variation were obtained, while the contact force was not recorded. For more details about the experiments, one can refer to the authors' previous paper [7].

(a)

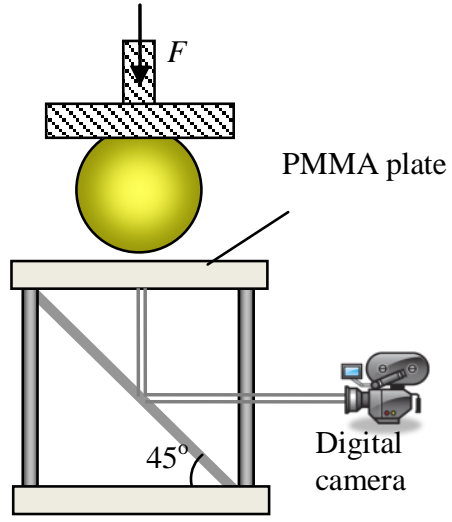

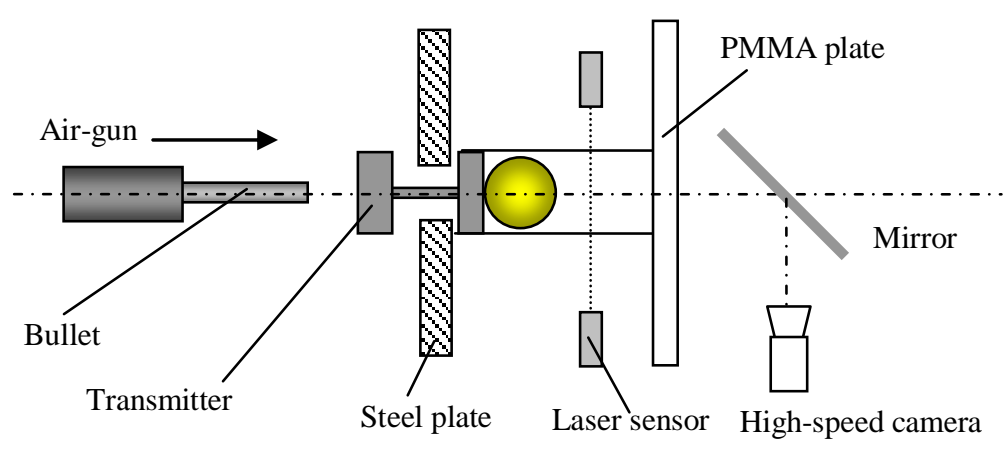

(b)

Figure (1): Experimental setup: (a) for quasi-static tests; (b) for impact tests 
(a)

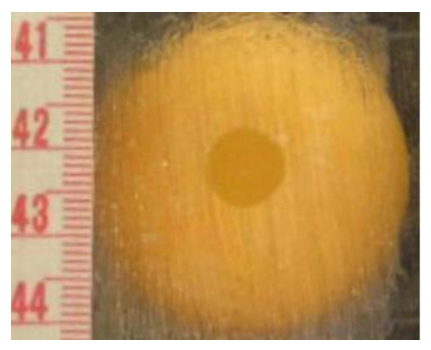

(b)

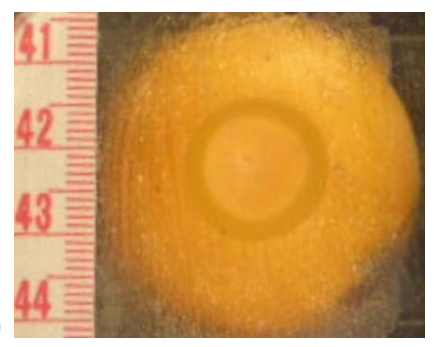

(c)

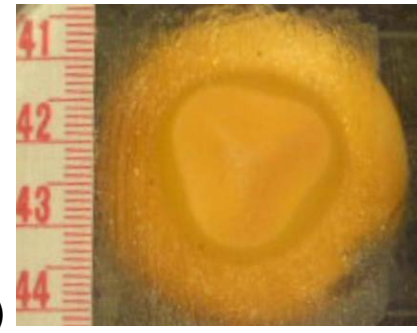

Figure (2): Local contact regions in quasi-static tests: (a) pre-buckling stage; (b) post-buckling stage

Some representative pictures of the contact region during the quasi-static tests are shown in Fig. 2. It is seen that the shell contacted the plate in a circular flattened region during the prebuckling stage, while in the post-buckling stage, the middle part of the contact region departed from the plate, indicating a snap-through buckling. With the compression progressed, the contact ring expanded and finally changed to a non-symmetric shape as shown in Fig. 2(c).

Four typical load-displacement curves due to quasi-static compression tests are plotted in Fig. 3(a). The deformed shells recovered their original shape in Tests 1 and 3, while the deformed shapes in Tests 2 and 4 were irrecoverable. It is found that there are two transition points on the loading curves, corresponding to the transitions between the three typical contact stages. Measured from the photos taken during the tests, the relationship between the displacement of the crosshead and the outer/inner diameters of the contact ring is obtained, as plotted in Fig. 3(b). The inner diameter is zero during the initial contact stage, which indicates a flat contact region. After the snap-through, the inner diameter of the contact ring increased rapidly and the ring's width became constant after the first transition point. The observed outer contact diameter is slightly smaller than that predicted by rigid-plastic

model.

(a)

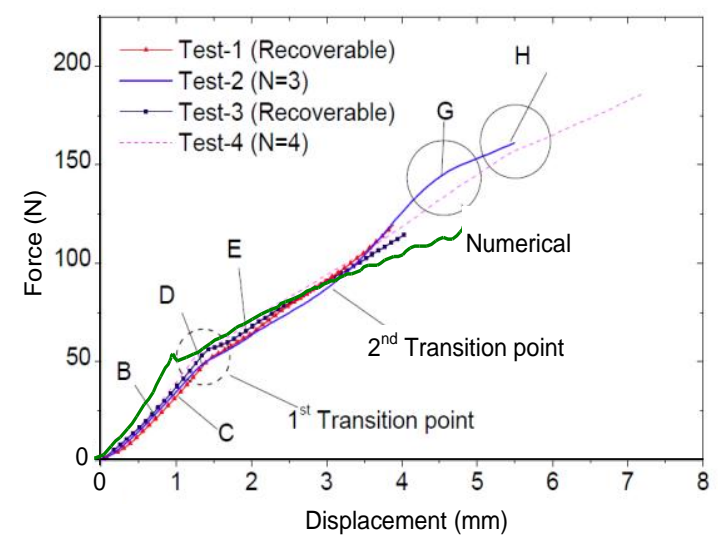




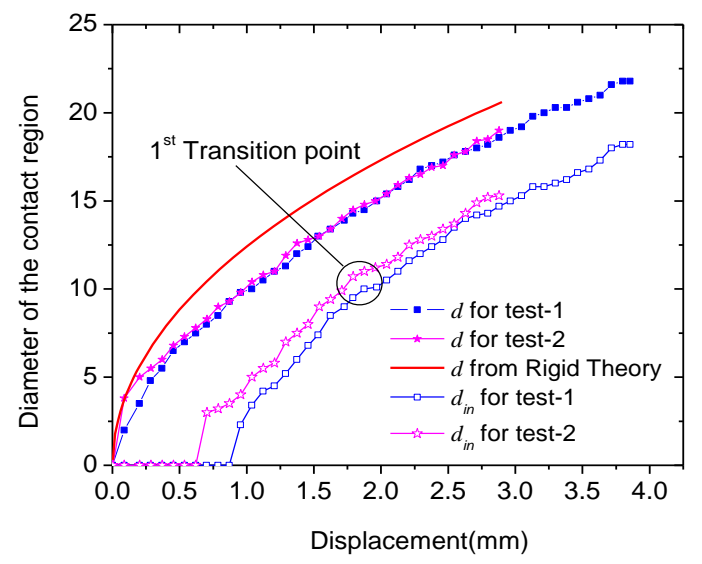

(b)

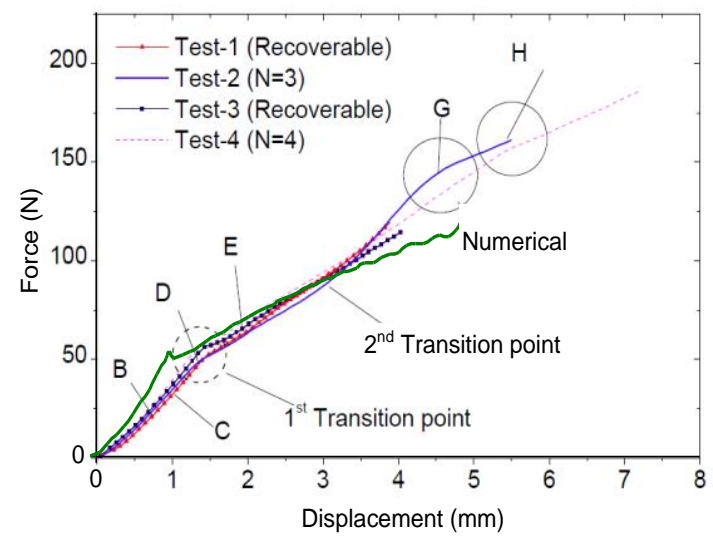

(a)

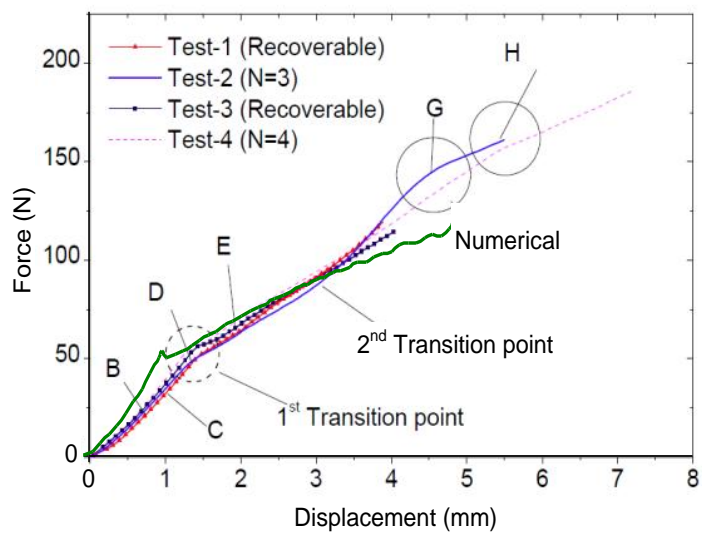

(a)

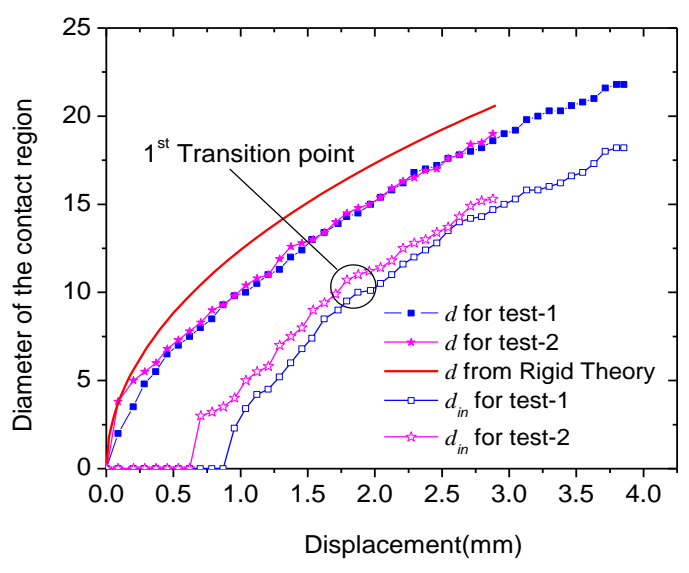

(b)

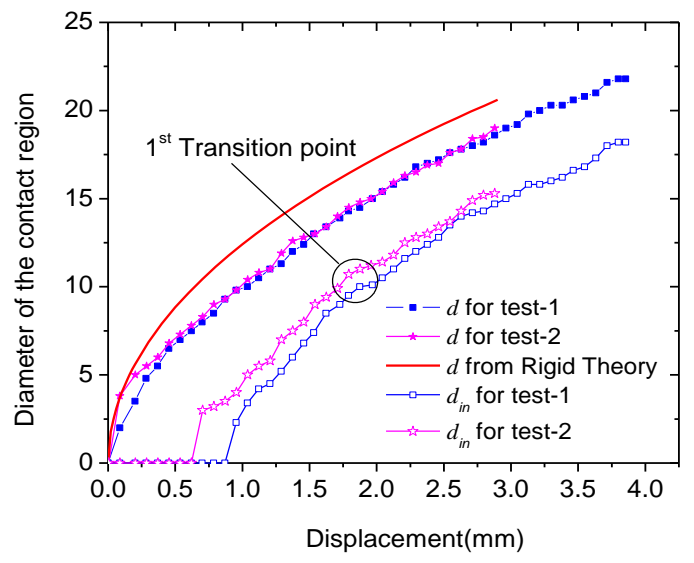

(b) 


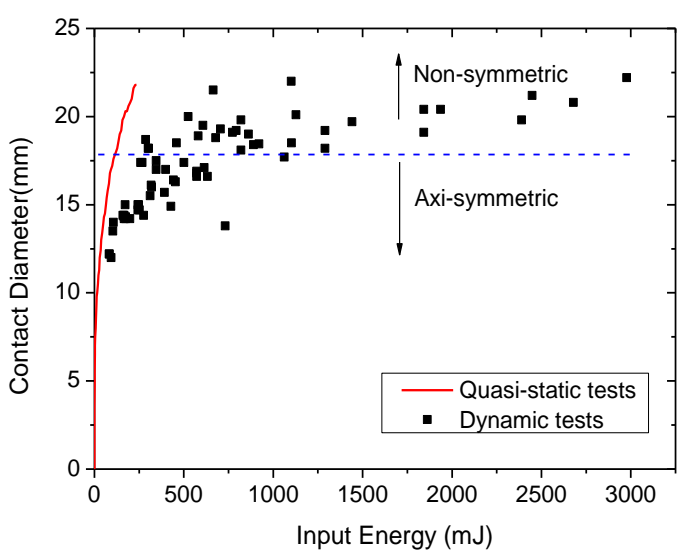

(c)

(d)

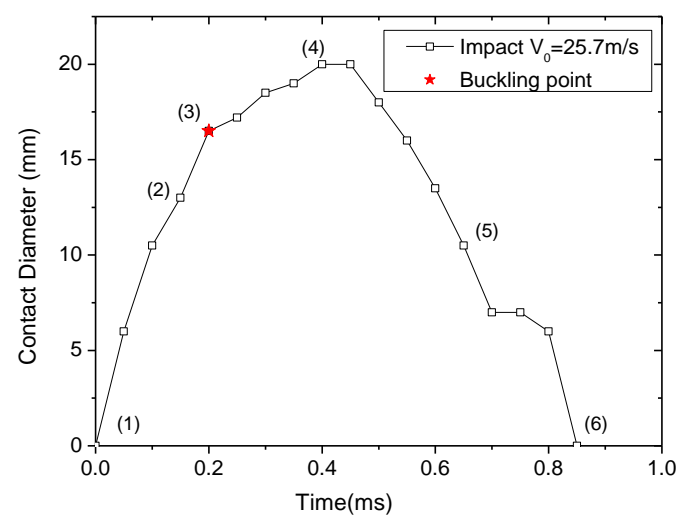

Figure (3): Experimental results: (a) Load-displacement curve under quasi-static compression; (b) Variation of contact diameter under quasi-static compression;

(c) Relation between the max contact diameter and the input energy; (d) Variation of contact diameter with time during impact

Fig. 3(c) depicts the maximum contact diameter as a function of the input energy, showing that this diameter increases with the increase of input energy. One can see that the maximum contact diameter remains limited for large impact energies. Non-symmetric deformation buckling modes were mostly observed for contact diameter larger than $18 \mathrm{~mm}$, which agrees well with the observations in quasi-static tests. However, the impact energy required for the same contact diameter is much larger than that required by the quasi-static compression. A typical contact diameter history for the impact velocity $V_{0}=25.7 \mathrm{~m} / \mathrm{s}$ is plotted in Fig. 3(d), showing that the maximum contact diameter was $20 \mathrm{~mm}$, and the duration of entire impact event was about $0.8 \mathrm{~ms}$. Besides, 
by checking the high speed video, it is revealed that the buckling happened at $d=17 \mathrm{~mm}$, which was larger than that observed in the quasi-static tests.

\section{Numerical simulations \\ 3. Numerical simulations \\ 3. Numerical simulations}

Since the contact force was not measured during the impact tests, numerical simulations are carried out to reveal the dynamic effects on the force and on the development of the deformed profiles during the snap-through process. Numerical simulations are performed using FE code ABAQUS/Standard and Explicit. The models for quasi-static and impact simulations are illustrated in Fig. 4.

Similar to the experimental set-up, only a part of the spherical shell shown in Fig. 4(a) (excluding the pre-buckled part) is analysed in the quasi-static simulations and the plate is modelled as a rigid body. The shell is fixed at its bottom, while a rigid plate moves downwards compressing the shell from the top. In the impact simulations, the whole shell is modelled and impinged onto a fixed rigid plate with a velocity $V_{0}$, as shown in Fig. $4(\mathrm{~b})$. For both simulations, shell elements S4R (4-node with reduced integration) are used to model the spherical shell. Surface-to-surface contact without friction between the shell and plate is adopted. The total element numbers of quasi-static and impact models are 8571 and 9025 , respectively. Denser mesh is used within the region where large deformation is expected.

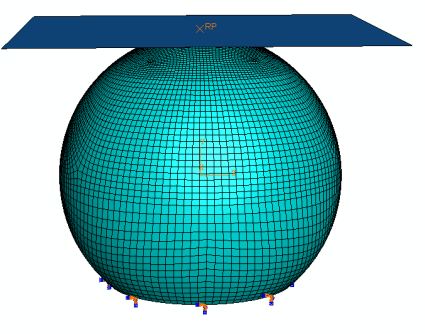

(a)

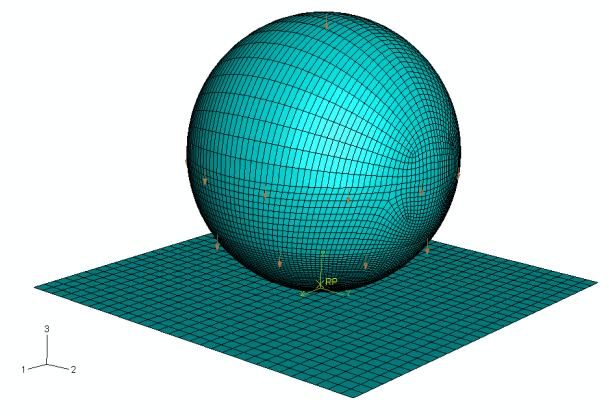

Figure (4): FEM models:

(a) for quasi-static compression; (b) for impact tests 


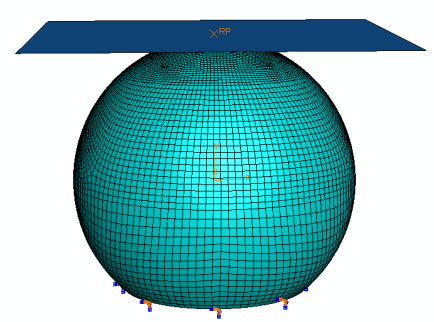

(a)

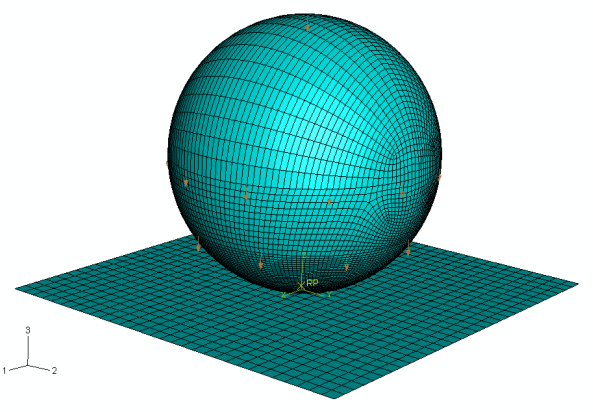

(b)

Figure (4): FEM models:

(a) for quasi-static compression; (b) for impact tests

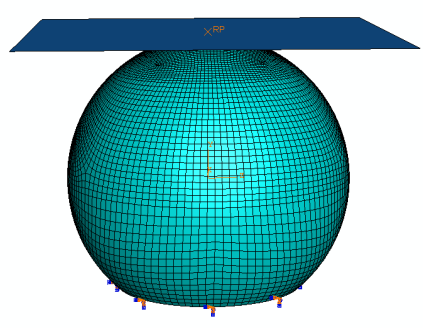

(a)

(b)

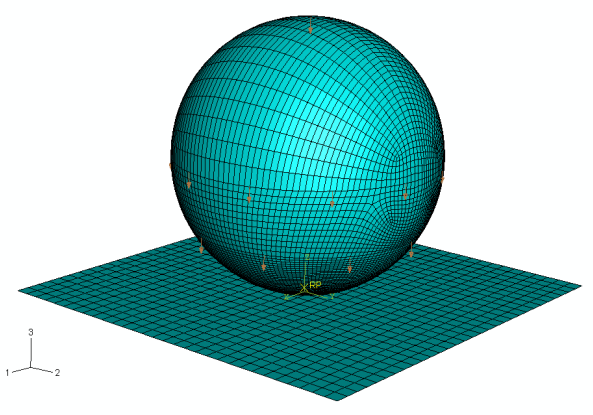

Figure (4): FEM models:

(a) for quasi-static compression; (b) for impact tests

Figure (4): FEM models: (a) for quasi-static compression; (b) for impact tests

Figure (4): FEM models: (a) for quasi-static compression; (b) for impact tests 


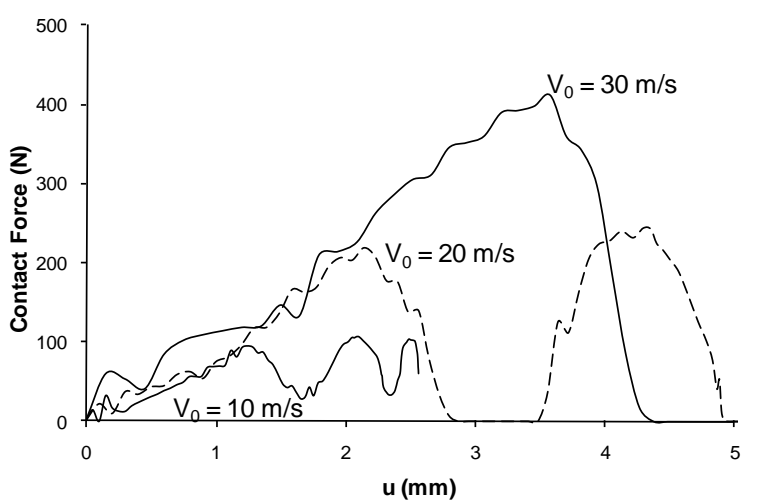

(a)

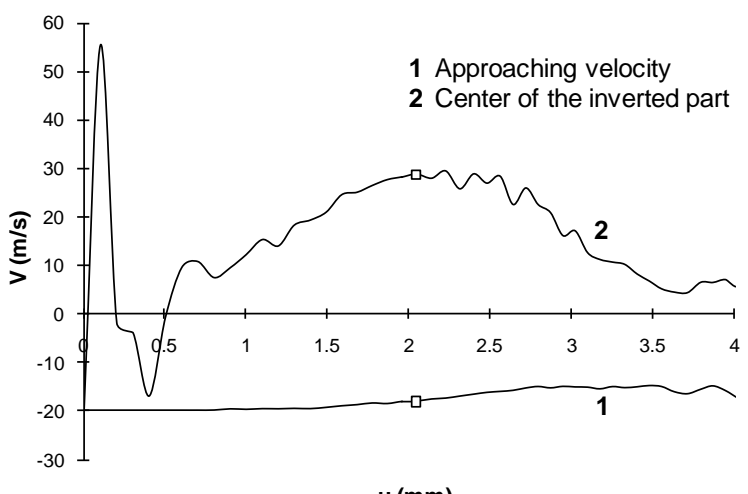

(b)

$\mathrm{u}(\mathrm{mm})$

Figure (5):

Numerical results for impact: (a) loading curves; (b) velocity history

Figure (5): Numerical results for impact: (a) loading curves;

(b) velocity history

Figure (5): Numerical results for impact: (a) loading curves;

(b) velocity history

Comparison between the force-displacement histories in four quasi-static tests and the numerical predictions assuming elastic properties of the shell is shown in Fig. 3(a). The agreement between the responses up to $u \approx 3.5 \mathrm{~mm}$ suggests that no significant inelastic deformation occurs; hence, the shell material can be considered as entirely elastic for relatively small displacements.

The results for impact loading with $V_{0}=10 \mathrm{~m} / \mathrm{s}, 20 \mathrm{~m} / \mathrm{s}$ and $30 \mathrm{~m} / \mathrm{s}$ are analysed to explore the dynamic effects on the deformation process. A principal effect of the increased impact velocity observed from the numerical simulation is that the contact force between the shell and the flat surface increases significantly as shown in Fig. 5(a). Besides, no obvious flattening appears under impact, unlike what happens in the pre-buckling phase of the shell 
deformation under static compression; instead, a small central region with inverted spherical-like surface is observed (Fig. 6). The rapid increase of the velocity (Fig. 5(b)) in the direction opposite to the loading direction acts at a small region of the shell within a short time period, causing the initial deformation similar to the response to a point load. For larger displacements $u$, however, the dynamic invert displacements are smaller than the corresponding static ones as shown in Fig. 6, which can explain that in the dynamic tests, the buckling diameter was larger than that in the quasi-static compression.

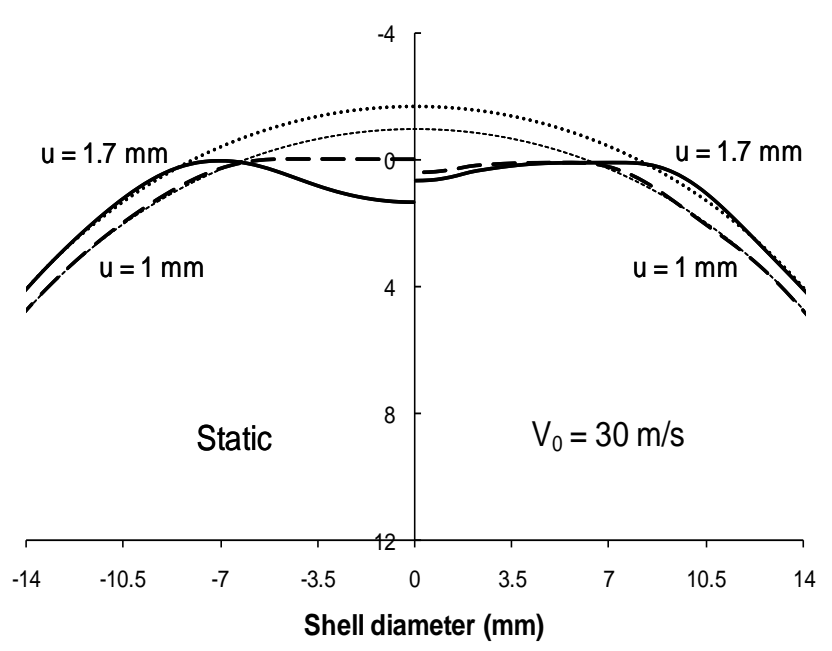

Figure (6): Deformed shapes of

the shell under quasi-static and dynamic loading:

Figure (6): Deformed shapes of the shell under quasi-static and dynamic loading:

Figure (6): Deformed shapes of the shell under quasi-static and dynamic loading: left: static loading; right: $V_{0}=30 \mathrm{~m} / \mathrm{s}$

\section{Theoretical analysis}

In order to deepen our understanding of the dynamic effects, theoretical models are developed for both quasi-static and impact processes using an energy approach. Based on the observations in the experiments, only the first two deformation stages are analysed here, i.e., the local flattening of the shell and the subsequent snap-though into an axi-symmetric mode. It is assumed that the deformed shape is characterised by large displacements (compared with the thickness of the shell) but relatively small strains which allows an assumption of an entirely elastic behaviour. 
(a)

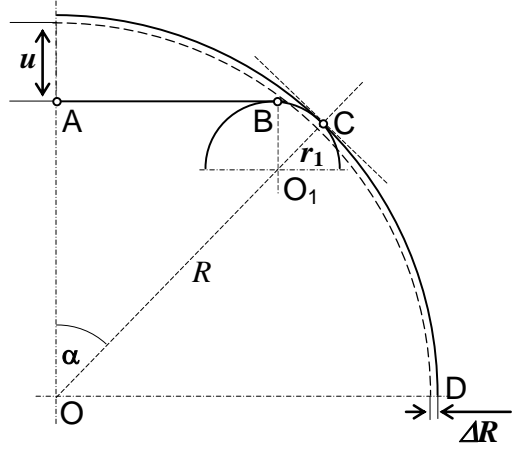

(b)

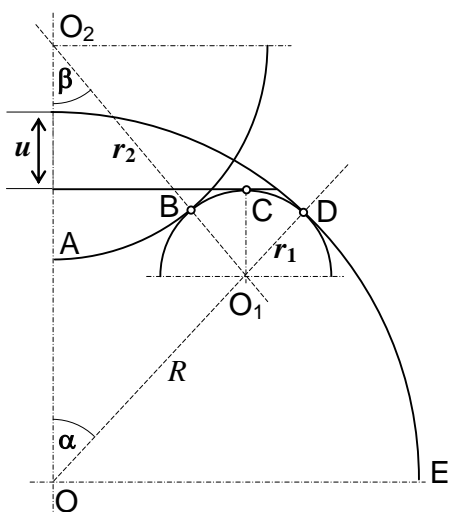

(c)

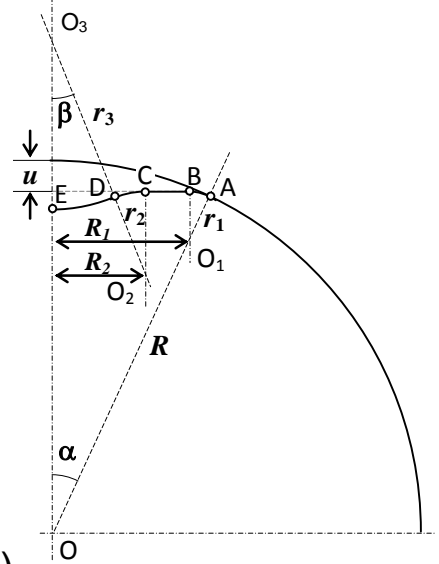

Figure (7): Theoretical model: (a) 1-r model for pre-buckling stage;

(b) 2-r model for post-buckling stage; (c) 3-r model for impact

Fig. 7(a) illustrates the geometry of the model for the pre-buckling stage in quasi-static compression, in which the deformed shape is composed of a spherical part, a toroidal and a flat surface. It is assumed that the shell is inextensional along the meridian The shape parameters in this deformation stage are the normal curvature $r_{1}$ of the torus surface representing the knuckle and $\Delta R$, which takes into account the circumferential expansion of the shell. On the other hand, two shape parameters describing the post-buckling phase are curvature radii $r_{1}$ and $r_{2}$ as shown in Fig. 7(b). The geometric relation in the pre-buckling stage can be expressed as

$$
(\pi / 2-\alpha)(R+\Delta R)+\alpha r_{1}=\pi R / 2
$$

while the relationship

$$
(\alpha+\beta) r_{1}+\beta r_{2}=\alpha R
$$

between the characteristic parameters is used for the 2-r model, which describes the postbuckling stage. If the displacement of the plate $u$ is given, the independent parameter $r_{1}$ and the proportional relationships $q=\Delta R / r_{1}$ and $q=r_{2} / r_{1}$ describe uniquely the development of the deformed shapes for the two stages of deformation.

According to Lagrange variational principle, the real deformed shape, $S^{\prime}$, should make the functional

$$
W=U_{D}\left(S^{\prime}\right)-W_{E}\left(S^{\prime}\right)
$$

to be stationary, i.e., its variation $\delta W=0$, which means

$$
W=d U_{D}\left(S^{\prime}\right)=d W_{E}\left(S^{\prime}\right)
$$

and the contact force is obtained

$$
F(u)=\frac{d W_{E}}{d u}=\frac{d U_{D}(u, \bar{q})}{d u} \approx \frac{\Delta U_{D}(u, \bar{q})}{\Delta u} .
$$


The comparisons between the theoretically and numerically deformation energy are plotted in Fig. 8(a). It can be seen that the analytical predictions agree well with the numerical results for displacement $u<3 \mathrm{~mm}$. Details of the energy variation during the pre-buckling stage are shown in Fig. 8(b), in which the intersection of the pre-buckling and post-buckling curves marks the transition point between the two deformation stages. It is found that the theoretical transition happens at $u=1.1 \mathrm{~mm}$.

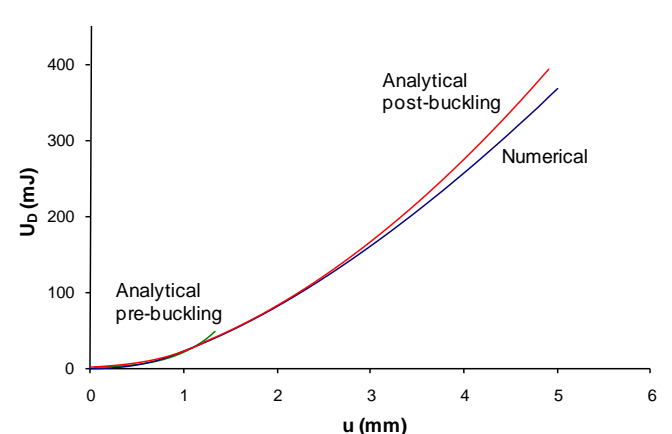

(a) loading curves; (b) determination of the transition point

As shown in Fig. 5(a), the contact force during the impact event is larger than that in the quasi-static compression, which is attributed to the inertia effect of the shell. Besides, it is found from the deformed shape of the shell, that there is no perfect flat contact area in under impact loading as shown in Fig. 6. Therefore, the deformation models used for the static behaviour of the shell are modified to reflect the observed deformation pattern. A new 4region model is proposed to analyse the dynamic snap-through behaviour, which starts at $t=$ 0 . The deformed region of the model in Fig. 7(c) contains one inverted spherical cap, two regions containing parts of torus surfaces with different normal curvatures and a flat part. The condition of in-extensional meridian leads to

$$
\beta\left(r_{2}+r_{3}\right)+\alpha r_{1}+\overline{B C}=\alpha R
$$

For this model, the parameters for the deformed shape are $p(u)=r_{2}(u) / r_{1}(u)$ and $q(u)=r_{3}(u) / r_{1}(u)$.

According to the extended Hamiltonian principle, the total change of the system energy is equal to the rate at which the non-conservative work is done on the system

$$
\frac{d U_{T}}{d t}=\frac{d W_{E}}{d t}=F(t)
$$

where $\delta U_{T}=\sum_{i=1}^{n}\left(m_{i} \frac{d^{2} \xi_{i}}{d t^{2}}\right) \cdot \delta \xi_{i}+\delta U_{D}$. The contact force is obtained incrementally as

$$
F\left(u_{i+1}\right)=\frac{\Delta W_{E, i+1}(u, \bar{p}, \bar{q})}{\Delta u}=\frac{\Delta U_{T}\left(u_{i+1}, \bar{p}_{i+1}, \bar{q}_{i+1}\right)}{\Delta u} .
$$


(a)

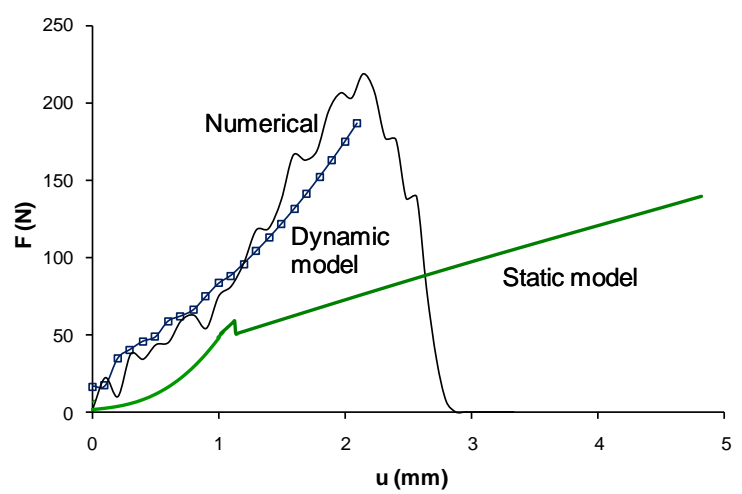

(b)

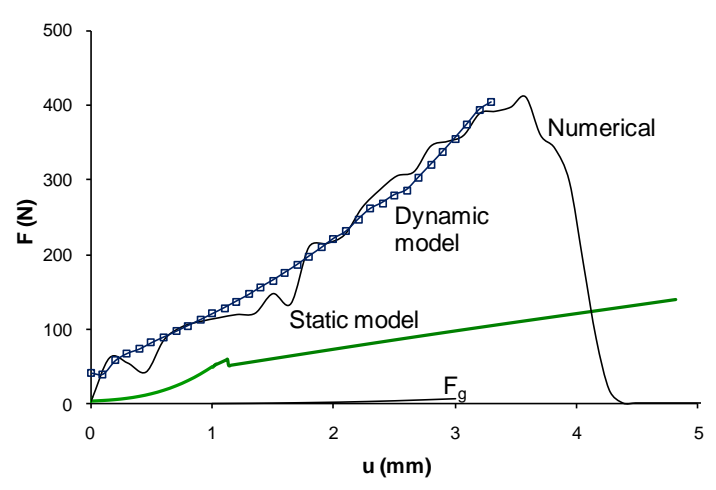

Figure (9): Comparison of contact force: (a) $V_{0}=20 \mathrm{~m} / \mathrm{s} ;$ (b) $V_{0}=30 \mathrm{~m} / \mathrm{s}$

The comparison between the static and dynamic contact forces shown in Fig. 9 indicates a significant increase of the contact force in dynamic cases, especially under higher impact velocity. The theoretical curves are calculated for displacement intervals $\Delta u=h / 2=0.2 \mathrm{~mm}$ and the corresponding points are marked on the curves. A reasonable agreement between the numerical and theoretical predictions is observed.

\section{Concluding remarks}

The analysis of the quasi-static and dynamic buckling of a thin-walled spherical shell compressed on a flat rigid surface has revealed some characteristic differences between the two types of response. The minimum deformation energy is associated with the static snapthrough response. In this case, the initial flattened region snaps-through and the curvature of the inverted cap approaches the curvature of the original shell with an opposite sign while relatively narrow knuckle connects the inverted cup with the undeformed part of the shell. In the way of contrast, no flat part develops before the snap-through buckling in the dynamic response. The width of the dynamically developed circumferential knuckle is larger due to the influence of the local inertia thus clarifying a source of the 'delay' of the dynamic snapthrough observed in the impact experiments. The development of the snap-through region restrained by the inertia forces is characterised by an increase of the deformation energy since the minimum value, which has been obtained in the static case cannot be achieved. The static and dynamic deformation energies predicted by the numerical simulations and the theoretical model are presented.

It should be noted that the present analysis considers only the initial deformation phase when despite the large impact energy the deformation pattern remains axi-symmetric. The experimental results show that even under impact velocities as high as $45 \mathrm{~m} / \mathrm{s}$ the deformation of the ping pong balls is entirely recoverable, which means that the developed non-symmetric deformations remain small so that the total displacements along the direction of loading are not very large. This phenomenon cannot be fully explained by the inertia effects, as considered in this study. Presumably, the strain-rate sensitivity of the ball material would be responsible for the possible increase of the elastic modulus of the ball material thus leading to a further increase of the local shell stiffness. 


\section{Acknowledgements:}

The study in this paper is supported by the National Natural Science Foundation of China under Key Project No. 10532020. Its financial assistance is gratefully acknowledged.

\section{References:}

[1] D.P. Updike, A. Kalnins, Axisymmetric behavior of an elastic spherical shell compressed between rigid plates. ASME Journal of Applied Mechanics, 92 (1970) 635-640.

[2] D.P. Updike, A. Kalnins, Axisymmetric postbuckling and nonsymmetric buckling of a spherical shell compressed between rigid plates. ASME Journal of Applied Mechanics, 94 (1972) 172-178.

[3] R. Kitching, R. Houlston, W. Johnson, Theoretical and experimental study of hemispherical shells subjected to axial loads between flat plates. International Journal of Mechanical Sciences, 17 (1975) 693-703.

[4] A.J. Morris, C.R. Calladine, 'The local strength of a spherical shell loaded radially through a rigid boss', in: I. Berman (Ed.), Proc. First Conf. on Pressure Vessel Technology, ASME, Part 1, 1969, pp. 35-44.

[5] N.K. Gupta, N. Mohamed Sheriff, R. Velmurugan, Experimental and numerical investigations into collapse behaviour of thin spherical shells under drop hammer impact, International Journal of Solids and Structures 44 (2007) 3136-3155.

[6] H.H. Ruan, Z.Y. Gao, T. X. Yu, Crushing of thin-walled spheres and sphere arrays. International Journal of Mechanical Sciences, 48 (2006) 117-133.

[7] X.W. Zhang, R. Fu, T.X. Yu, Experimental study on static/dynamic local buckling of ping pong balls compressed onto a rigid plate, International Conference of Experimental Mechanics 2009, Singapore, 18-20 November, 2009. 